\title{
FK Comae and the Evolution of Close Binaries
}

\author{
DAVID P. HUENEMOERDER \\ Jet Propulsion Laboratory \\ LAWRENCE W. RAMSEY \\ Pennsylvania State University \\ DEREK L. BUZASI \\ Johns Hopkins University \\ and \\ HAROLD L. NATIONS \\ Franklin and Marshall University
}

September 10, 1991

\begin{abstract}
.
FK Com is an enigma among active cool stars. A rotationally driven magnetic dynamo is an explanation for activity. Youth and binarity are two causes of rapid rotation, but FK Com is old, single, and rotating near breakup. Much studied optical and ultraviolet data have not revealed the cause of its unusual activity. It is an important object in a more complete understanding of close binary star evolution and of the dynamo theory of magnetic activity. In 1989, we executed a coordinated ultraviolet and intensive optical spectroscopic and photometric campaign to better elucidate its characteristics. Data relevant to its evolutionary status, namely its radial velocity variations, will be discussed here, while an in depth study of the chromospheric activity will be deferred to a more detailed publication.
\end{abstract}

\section{Introduction}

The Solar activity paradigm has been long in use for interpretation of phenomena in cool stars, ranging from pre-main-sequence stars, active dwarfs and dwarf binaries (BY Dra stars), to evolved binaries (RS CVn stars). Phenomena which have been invoked to explain photometric modulation, various emission lines, and X-ray emission are cool spots, plage, prominences, chromospheres, transition regions, and coronae, in analogy with the Sun. Rapid rotation due to youth or tidal coupling is the common feature among the diverse mix of active stars. However, the details of the conversion of magnetic energy into thermal heating is still a subject of intense investigations. As extreme cases often establish the rule or its exceptions, FK Com has been a natural target. Its rotation rate of $v \sin i=160 \mathrm{~km} \mathrm{~s}^{-1}$ (Buzasi, 1987) is extreme. An additional puzzle is its lack of a companion, known by lack of significant radial velocity variations (McCarthy and Ramsey 1984). This leads to the hypothesis that FK Com is a recently coalesced binary, though Walter and Basri (1982) suggested that there is an extremely low mass companion and an accretion stream. Either case puts FK Com in an interesting evolutionary state.

\section{Basic Parameters}

Previous published values using surface fluxes or distance-dependent parameters have had to use fairly uncertain quantities. Recent work has improved some of these. Eggen and Iben (1988) have identified FK Com as a member of the old disk 
group, HR 1614. From their Figure 4, $M_{V}$ is estimated to be about 1.5, which when combined with the mean visual magnitude of 8.2 yields a distance of $219 \mathrm{pc}$. Our optical spectroscopic campaign over eight nights with an echelle spectrograph gave us an improved rotational velocity of $162 \pm 4 \mathrm{~km} \mathrm{~s}^{-1}$. Combined with the photometric period of $2.4 \mathrm{~d}$ (Chugainov 1976) yields $R \sin i=7.7 R_{\odot} \pm 0.2$. Since the light-curve is never flat-topped, we infer that there is moderate inclination. Given a mass of about $1.5 M_{\odot}$ implied by Eggen and Iben, the inclination must be greater than $55^{\circ}$ to have a velocity less than or equal to break-up. Adopting the median implied $\sin i$ of 0.9 gives a radius of 8.6 $R_{\odot}$. Applying this along with the visible flux at the Earth and a surface flux calculated from the Barnes-Evans relationship (Barnes, Evans, and Moffett 1978), using $(V-R)=0.75$ from Bianchi and Grewing (1985), gives a distance of $215 \mathrm{pc} \pm 5$. The Barnes-Evans visual and red surface fluxes can also be used to calculate the black-body equivalent effective temperatures, and give $T_{\text {eff }}=5800 \mathrm{~K}$. This is consistent with the Bianchi and Grewing value of $6000 \mathrm{~K}$ estimated from IUE continuua. We can hence estimate a bolometric luminosity of $2.9 \times 10^{35} \mathrm{ergs} \mathrm{s}^{-1}$. Hence we now have independent, consistent, and believable fundamental parameters for FK Com.

\section{FK Com and Binary Evolution}

There are two basic questions to be answered regarding the nature of FK Com: Is it really a single star? How did it get that way? We can address the first in a straightforward way by measuring radial velocities. For the second, we have incomplete models and much speculation.

In our eight-night run we collected 51 spectra at a resolution of $\lambda / \Delta \lambda=12,000$ with nearly complete spectral coverage. These data provide many photospheric absorption lines with which to determine the radial velocities. This was done by fitting a standard star's template spectrum to those of FK Com by artificially rotationally broadening it (using a broadening function appropriate to a spherical, rigidly rotating body, which is a leap of faith), and by shifting the template in velocity. This was done for eight spectral orders which had no significant chromospheric emission lines (primarily Balmer and Ca II lines), or strong telluric lines, covering the wavelength region between $\mathrm{H} \alpha$ and $\mathrm{H} \beta$. While there were some systematic zero-point errors between different echelle orders preventing a good determination of the systemic velocity, the form of the velocity variations was the same in each order. Thus they were scaled to a common mean to give a relative radial velocity curve. These data are shown in Figure 1. The error-bars reflect the dispersion among echelle orders after shifting to a common mean. The one-sigma uncertainty is about $1 \mathrm{~km} \mathrm{~s}^{-1}$, while the amplitude of variation is about $5 \mathrm{~km} \mathrm{~s}^{-1}$. This amplitude is about equal to the upper limit set by McCarthy and Ramsey (1984), and all their arguments regarding the nature of a companion apply. However, the form of the variations here is definitely non-sinusoidal, and is not the signature of a binary companion. Sample profiles and the fitted standard star spectrum for two different rotational phases are shown in Figure 2. It can be plainly seen that one of them has a central bump. We attribute this to the signature of a dark photospheric spot, resolved within the rotational broadening profile. The amplitude of the visual light curve was about 0.1 


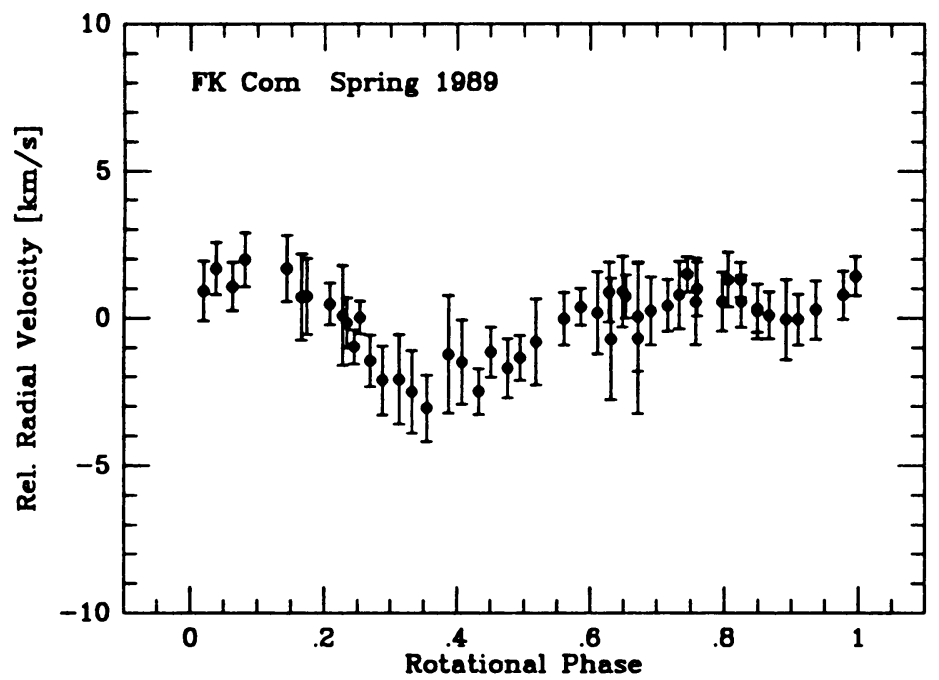

Fig. 1. FK Com relative radial velocities

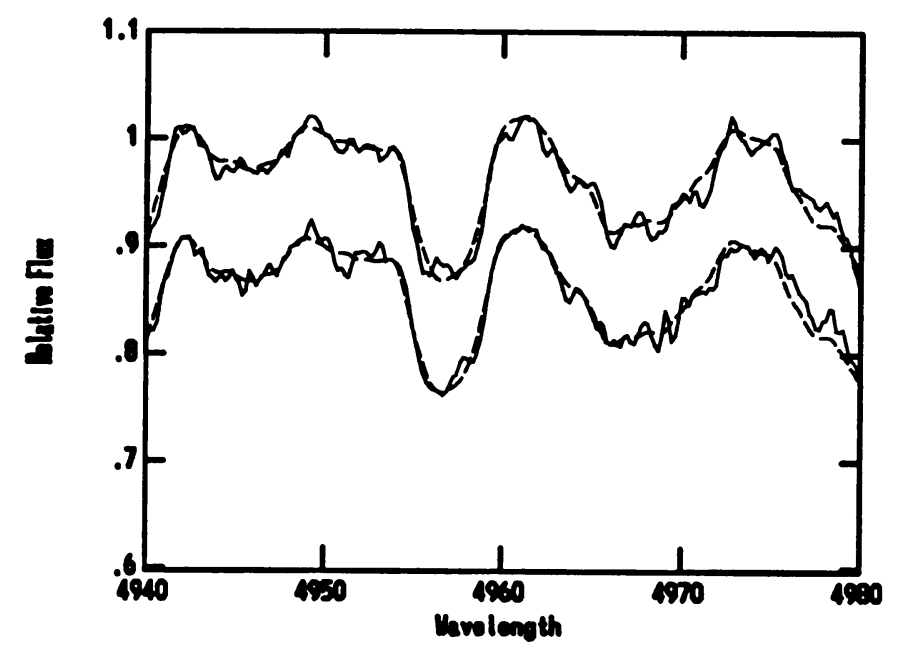

Fig. 2. Line profiles: solid, FK Com; dashed, fitted standard

mag at this time, and the behavior of the bumps corresponds reasonably well with the photometric brightness and presumed spot visibility. This, however, makes the radial velocity a model-dependent parameter. There could still be some residual sinusoidal variation masked by the spot, but such a determination requires a spot model and the associated theoretical line profiles. In any case, the upper limit to the radial velocity due to orbital motion can probably be reduced to 2 or $3 \mathrm{~km} \mathrm{~s}^{-1}$ and further constrain the characteristics of any purported secondary.

FK Com is often proclaimed to be a coalesced binary, a result of a scenario given by Webbink (1976) as the final state of a W UMa system. Theoretical models show how stars may undergo mass loss and transfer to become an Algol system 
(Tout and Eggleton, 1988), or how the formation of a double-core may eject an envelope (Taam and Bodenheimer, 1989). Models of low-mass double-core or fully coalesced systems do not yet exist. Such would be extremely valuable, particularly if they predicted observable radial velocity excitations of the outer envelope. There are few known FK Com-type objects. Given the high frequency of binarity, and the large fraction of those which are W UMa stars, either coalescence rarely happens, or the product is extremely short-lived. We are still under the burden of proof to actually show that the coalescence scenario is correct for FK Com. There are some intriguing objects - cores of planetary nebulae - which bear resemblances to FK Com (Bond, 1991). Studies of these may indeed link them to FK Com and vindicate the coalescence hypothesis.

\section{Atmospheric Activity}

As a preview of work in preparation for publication, we note some interesting aspects of the atmospheric activity. $\mathrm{H} \alpha$, while $1200 \mathrm{~km} \mathrm{~s}^{-1}$ broad at the base, has its position modulated in velocity by $v \sin i$. The whole line, as measured in the centroid or wing locations, oscillates at the rotational period. Its breadth is not due to extended, corotating, magnetically supported structures (they could still be extended, but towards the poles, and not beyond one stellar radius from the rotation axis.) $\mathrm{He} \mathrm{I} \mathrm{D}_{3}$ shows a strong absorption core and emission wings, both of which are variable. The line is typically not present in either emission or absorption in inactive stars of similar spectral type. Solar $D_{3}$ is limb-brightened except under coronal holes. We interpret the high excitation of helium in FK Com as a result of coronal X-ray radiation, and its variations as due to structure in the corona.

\section{References}

Barnes, T.G., Evans, D.S., and Moffett , T.J., 1978, MNRAS, 183, 285.

Bianchi, L., and Grewing, M., 1985, $A \& A, 283,200$.

Bond, H., 1991, private communication.

Buzasi, D.L., 1987, in Cool Stars, Stellar Systems, and the Sun, Proceedings of the Fifth Cambridge Workshop, edited by J. Linsky and R. Stencel, p. 518.

Chugainov, P. F., 1976, Izv. Krymsk. Ap. Obs., 54, 89.

Eggen, O.J., and Iben, I., 1988, in Progress and Opportunities in Southern Hemisphere Optical Astronomy, ed. V.M. Blanco and M.M. Phillips, A.S.P. Conf. Ser., 1, 239

McCarthy, J.K., and Ramsey, L.W., 1984, Ap.J., 283, 200.

Taam, R.E., and Bodenheimer, P., 1989, Ap.J., 337, 849.

Tout, C.A., and Eggleton, P.P., 1988, Ap.J., 334, 357.

Walter, F.M., and Basri, G., 1982, Ap.J., 260, 735.

Webbink, R.F., 1976, Ap.J., 209, 829.

Acknowledgements: The research described in this paper was carried out by the Jet Propulsion Laboratory, California Institute of Technology, under a contract with the National Aeronautics and Space Administration. 